\title{
Notes on the BMS group in three dimensions: $I$. Induced representations
}

\author{
Glenn Barnich and Blagoje Oblak \\ Physique Théorique et Mathématique, \\ Université Libre de Bruxelles and International Solvay Institutes, \\ Campus Plaine C.P. 231, B-1050 Bruxelles, Belgium \\ E-mail: gbarnich@ulb.ac.be, boblak@ulb.ac.be
}

ABSTRACT: The Bondi-Metzner-Sachs group in three dimensions is the symmetry group of asymptotically flat three-dimensional spacetimes. It is the semi-direct product of the diffeomorphism group of the circle with the space of its adjoint representation, embedded as an abelian normal subgroup. The structure of the group suggests to study induced representations; we show here that they are associated with the well-known coadjoint orbits of the Virasoro group and provide explicit representations in terms of one-particle states.

Keywords: Conformal and W Symmetry, Space-Time Symmetries, AdS-CFT Correspondence, Classical Theories of Gravity

ARXIV EPRINT: 1403.5803

\footnotetext{
${ }^{1}$ Research Director of the Fund for Scientific Research-FNRS Belgium.

${ }^{2}$ Research Fellow of the Fund for Scientific Research-FNRS Belgium.
} 


\section{Contents}

1 Introduction 1

2 Induced representations of semi-direct products 4

2.1 Semi-direct products 4

2.2 Orbits and little groups 4

2.3 Induced representations 5

2.4 The case of the adjoint action 6

$\begin{array}{lll}2.5 & \text { Remarks on projective representations } & 7\end{array}$

3 The Poincaré group in three dimensions $\quad 7$

$\begin{array}{lll}3.1 & \text { Double cover of the Poincaré group } & 7\end{array}$

3.2 Induced representations of the Poincaré group 9

4 Virasoro algebra and group $\quad 10$

$\begin{array}{lll}4.1 & \text { Virasoro algebra } & 10\end{array}$

$\begin{array}{ll}4.2 \text { Virasoro group } & 12\end{array}$

$5 \quad$ BMS group in three dimensions $\quad 13$

$\begin{array}{lll}5.1 & \text { Structure of the group and algebra } & 14\end{array}$

$\begin{array}{lll}5.2 & \mathrm{BMS}_{3} \text { as transformation group on } \mathscr{I} & 15\end{array}$

$\begin{array}{lll}5.3 \text { Poincaré subgroups of } \mathrm{BMS}_{3} & 15\end{array}$

6 Induced representations of $\mathrm{BMS}_{3} \quad 16$

$\begin{array}{lll}6.1 & \text { Little algebras } & 16\end{array}$

$\begin{array}{lll}6.2 & \text { Orbits and little groups } & 17\end{array}$

$\begin{array}{lll}6.3 & \text { Energy bounds } & 19\end{array}$

$\begin{array}{lll}6.4 & \text { Features of } \mathrm{BMS}_{3} \text { particles } & 20\end{array}$

$\begin{array}{lll}7 & \text { Open questions } & 22\end{array}$

\section{Introduction}

Motivation. Asymptotic symmetry groups play an important role in gravitational theories. From a modern perspective [1-3], this is because they are the global symmetry groups for the lower-dimensional, dual field theories. For example, the asymptotic symmetry group of a $d$-dimensional anti-de Sitter background is isomorphic to the conformal group in dimension $d-1$ [4-7]. From the point of view of symmetries, $d=3$ is then the most interesting dimension because the conformal algebra in dimension two is infinite-dimensional $[8,9]$. 
Historically, however, the first instance of an enhanced, infinite-dimensional symmetry group in general relativity appeared in the case of four-dimensional asymptotically flat spacetimes at null infinity, as shown by Bondi, van der Burg, Metzner and Sachs (BMS) [10-12]. There has recently been a lot of interest in this group in the context of gravitational scattering theory and soft graviton theorems [13-16] (see also [17] for a novel approach in the framework of conformal Carroll groups). In this framework, it is natural to expect that elementary particles occurring in scattering states should be classified by representations of the $\mathrm{BMS}_{4}$ rather than the Poincaré group [12, 18-20].

In line with the point of view taken in two-dimensional conformal field theories, one can also argue that physics should be constrained by infinitesimal symmetry transformations that are not necessarily globally well-defined. When adopting this viewpoint [21-23], the local symmetry algebra associated with $\mathrm{BMS}_{4}$ involves two copies of the Virasoro algebra and an adapted (larger) set of supertranslations.

Before studying this group head on, it is instructive to first understand what happens in three dimensions: the $\mathrm{BMS}_{3}$ group [24] is then again infinite-dimensional. While it is globally well-defined, its Lie algebra looks roughly like half of that of the local $\mathrm{BMS}_{4}$ algebra. More precisely, the Dirac brackets of its generators form the centrally extended semi-direct sum of the space of vector fields on the circle with its adjoint representation, embedded as an abelian ideal [25].

Hence, in an effort to extend some of the successes of holography for three-dimensional asymptotically anti-de Sitter spacetimes (see e.g. [26-29]) to flat space (see e.g. [22, 30-35]), the purpose of the present paper is to initiate the study of the representations of the $\mathrm{BMS}_{3}$ group and to show that, like in the conformal case, they are controlled by powerful, wellestablished results on the Virasoro group, which are readily available in the literature.

More precisely, we will consider induced representations. This is motivated by the fundamental theorem, due to Mackey (see e.g. [36-42]), stating that — under suitable regularity assumptions - the classification of all unitary irreducible representations of a semi-direct product group $H=G \ltimes A$, with $A$ abelian, can be obtained by

1. determining the set $\hat{A}$ of characters of $A$;

2. classifying all orbits in $\widehat{A}$ under $G$;

3. computing the stabilizers, the "little groups", of the orbits;

4. constructing a specific induced representation associated with every unitary irreducible representation of a given little group.

The origin of these general group-theoretic results is the celebrated work by Wigner [43] on the classification of the irreducible representations of the Poincare group.

The $\mathrm{BMS}_{3}$ and $\mathrm{BMS}_{4}$ groups that we are interested in here have precisely the semidirect structure required by the theorem. They fail, however, to satisfy all the regularity assumptions because either $A$, or both $A$ and $G$, are infinite-dimensional.

The semi-direct product structure of the globally well-defined $\mathrm{BMS}_{4}$ group in four dimensions has been clarified in $[44,45]$. Its induced representations have been constructed 
in $[46-51]$, and shown in $[52,53]$ to exhaust all unitary irreducible representations. These results imply, for instance, the absence of particles with continuous spin.

In three dimensions, the $\mathrm{BMS}_{3}$ group is the semi-direct product $\operatorname{Diff}^{+}\left(S^{1}\right) \ltimes_{\mathrm{Ad}}$ $\operatorname{Vect}\left(S^{1}\right)_{\text {ab }}$ of the group of orientation-preserving diffeomorphisms of the circle with its Lie algebra embedded as an abelian normal subgroup, so that both factors are infinitedimensional. The property that makes this semi-direct product special, and is shared by the Poincaré group in three dimensions - a subgroup of $\mathrm{BMS}_{3}$-, is that the non-abelian group $G$ acts on the abelian group $A$ through the adjoint action, $G \ltimes_{\text {Ad }} \mathfrak{g}_{\mathrm{ab}}$. In this case, the orbits relevant for induced representations coincide with the coadjoint orbits of $G$. The latter are endowed with a canonical symplectic structure and, upon quantization, play an important role in the representation theory of $G$ itself (see e.g. [54]). The main difference here is that, for representations of $G \ltimes$ Ad $\mathfrak{g}_{\mathrm{ab}}$, there is no need to quantize the coadjoint orbits. Their symplectic structure does nevertheless play an important role, as it provides an invariant measure needed for induced representations.

In the case of $\mathrm{BMS}_{3}$ and its central extension $\widehat{\mathrm{BMS}}_{3}$, induced representations are thus classified by coadjoint orbits of $\operatorname{Diff}^{+}\left(S^{1}\right)$ and of the Virasoro group $\widehat{\operatorname{Diff}^{+}}\left(S^{1}\right)$, which have been extensively studied in the literature (see e.g. [55, 56] and also [57-59] for discussions in the physics literature).

The $\mathfrak{b m s}_{3}$ algebra has also appeared in the framework of two-dimensional statistical systems in the form of an infinite-dimensional extension of $\mathfrak{a l t}_{1} \cong \mathfrak{i s o}(2,1)$ [60]. It is also isomorphic to the infinite-dimensional extension of the Galilean conformal algebra in two dimensions, $\mathfrak{g c a}_{2}$ [61-63]. The classification of induced representations of $\mathrm{BMS}_{3}$ is thus also applicable in these cases, and governs the physics of these problems.

A companion paper will be devoted to the coadjoint representation of $\widehat{\mathrm{BMS}}_{3}$, which coincides with the reduced phase space of three-dimensional asymptotically flat gravity. In particular, we will establish a direct connection between induced representations and the quantization of coadjoint orbits of $\widehat{\mathrm{BMS}}_{3}$.

Plan of the paper. The paper is organized as follows. In section 2, we briefly review relevant results on induced representations. This is followed by remarks on projective representations [64] (see e.g. [65, 66] for a summary). In section 3, we then apply the inducing construction to the example of the Poincaré group in three dimensions [67].

In section 4, we collect some remarks on the Virasoro algebra and group, following in particular [68-70] (see also [71] for a detailed analysis of a group with a structure similar to that of $\mathrm{BMS}_{3}$ ). These preliminaries are then used in section 5 to discuss the structure of the $\mathrm{BMS}_{3}$ group and of its extensions. We also review the realization of $\mathrm{BMS}_{3}$ as a transformation group on null infinity $\mathscr{I}$, and isolate its Poincaré subgroups.

In section 6 on induced representations of $\mathrm{BMS}_{3}$, we start by reviewing the Virasoro coadjoint orbits. We also discuss energy bounds, mostly following [72] to which we refer for a more complete and self-contained review (see also [69]). We then describe features of $\mathrm{BMS}_{3}$ particles, that is, induced representations of $\widehat{\mathrm{BMS}}_{3}$, by taking inspiration from the Poincaré group in three dimensions.

Finally, section 7 is devoted to an enumeration of open issues, some of which we hope to address elsewhere. 


\section{Induced representations of semi-direct products}

In this section we review the construction of induced representations for separable, locally compact topological groups. In particular, here and below "topological group" will mean that all these regularity assumptions are verified. It will also be understood that all group homomorphisms, actions and representations are continuous. We end the section with a brief review on projective representations.

\subsection{Semi-direct products}

Let $A$ and $G$ be two topological groups; elements of $G$ are denoted as $f, g$, etc. and elements of $A$ as $\alpha, \beta$, etc. For our purposes, $A$ is assumed to be an abelian additive vector group throughout, and therefore the group operation in $A$ is denoted as the addition +. Let GL $(A)$ be the linear group of $A$ and let $\sigma: G \rightarrow \operatorname{GL}(A), f \mapsto \sigma_{f}$ be a group homomorphism. The semi-direct product of $G$ and $A$ is the group

$$
H:=G \ltimes_{\sigma} A,
$$

whose elements are pairs $(f, \alpha)$, with a group operation given by

$$
(f, \alpha)(g, \beta):=\left(f g, \alpha+\sigma_{f} \beta\right) .
$$

Typical examples of groups of this form include the euclidean groups $\mathrm{SO}(n) \ltimes \mathbb{R}^{n}$ and the Poincaré groups $\mathrm{SO}(n-1,1)^{\uparrow} \ltimes \mathbb{R}^{n}$.

If $G$ is a Lie group, let $\mathfrak{g}$ be the corresponding Lie algebra, whose elements are written as $X, Y$, etc. Since $A$ is a vector group, it is isomorphic - as a vector space - to its Lie algebra, which is why we continue to denote elements of the latter by $\alpha, \beta$, etc. If $\operatorname{End}(A)$ is the commutator algebra of linear operators in $A$, the differential of the map $\sigma$ at the origin is the Lie algebra homomorphism $\Sigma: \mathfrak{g} \rightarrow \operatorname{End}(A), X \mapsto \Sigma_{X}$. Then the Lie algebra of the semi-direct product $H$ is the semi-direct sum $\mathfrak{h}=\mathfrak{g} \oplus_{\Sigma} A$. Writing elements of the latter as pairs $(X, \alpha)$, the Lie bracket in $\mathfrak{h}$ reads

$$
[(X, \alpha),(Y, \beta)]=\left([X, Y], \Sigma_{X} \beta-\Sigma_{Y} \alpha\right) .
$$

\subsection{Orbits and little groups}

Since $A$ is abelian, its unitary irreducible representations are necessarily one-dimensional. As a consequence, they are fully determined by their character, which can be written as

$$
\chi: A \rightarrow \mathbb{C}, \alpha \mapsto \chi(\alpha)=e^{i\langle p, \alpha\rangle},
$$

where the map $\langle p,\rangle:. A \rightarrow \mathbb{R}$ is a continuous homomorphism, assumed to be smooth in the case of Lie groups. The map $p$ belongs to $A^{*}$, the topological dual of $A$, which is thus in one-to-one correspondence with the set of inequivalent unitary irreducible representations of $A$. Elements of $A^{*}$ will be denoted by $p, q$, etc.

The action of $G$ on $A^{*}$ is the homomorphism $\sigma^{*}: G \rightarrow \mathrm{GL}\left(A^{*}\right), f \mapsto \sigma_{f}^{*}$ given by

$$
\left\langle\sigma_{f}^{*} p, \alpha\right\rangle:=\left\langle p, \sigma_{f^{-1}} \alpha\right\rangle
$$


For simplicity, we will use the notation $f \cdot p:=\sigma_{f}^{*} p$ below. The orbit of some $p \in A^{*}$ is then defined as the set

$$
\mathcal{O}_{p}:=\{f \cdot p \mid f \in G\} \subset A^{*} .
$$

The little group $G_{p}$ for this orbit is the stationary subgroup of $G$ for the element $p$ and the action $\sigma^{*}$,

$$
G_{p}:=\{f \in G \mid f \cdot p=p\} .
$$

By construction, the orbit $\mathcal{O}_{p}$ is a homogeneous space for the action $\sigma^{*}$ and, under suitable regularity assumptions, $\mathcal{O}_{p}$ is homeomorphic to the (left) coset space $G / G_{p}$. This identification can be made explicit by introducing a map

$$
g: \mathcal{O}_{p} \rightarrow G, q \mapsto g_{q},
$$

where $g_{q}$ is such that $g_{q} \cdot p=q$ for all $q \in \mathcal{O}_{p}$. One may then identify $q \in \mathcal{O}_{p}$ with the left coset $g_{q} G_{p} \in G / G_{p}$. The map $g$ is defined up to multiplication from the right by any map which sends $\mathcal{O}_{p}$ on $G_{p}$.

\subsection{Induced representations}

Generalities. Consider a unitary representation $\mathcal{R}: G_{p} \rightarrow \mathrm{GL}(\mathcal{E}): f \mapsto \mathcal{R}[f]$ of a given little group $G_{p}$ in a separable Hilbert space $\mathcal{E}$, equipped with a scalar product (.|.). A representation of the subgroup $H_{p}:=G_{p} \ltimes_{\sigma} A$ of $H$, where it is understood that $\sigma$ is restricted to $G_{p}$, is given by

$$
\mathcal{S}[(f, \alpha)]:=e^{i\langle p, \alpha\rangle} \mathcal{R}[f] \quad \forall(f, \alpha) \in H_{p}
$$

Let $\mu$ denote a $G$-invariant measure on the orbit $\mathcal{O}_{p}$ and consider the Hilbert space $\mathcal{H}$ of maps $\Psi: \mathcal{O}_{p} \rightarrow \mathcal{E}$ that are square-integrable with respect to this measure, the scalar product in $\mathcal{H}$ being defined as

$$
\langle\Phi \mid \Psi\rangle:=\int_{\mathcal{O}_{p}} d \mu(q)(\Phi(q) \mid \Psi(q)) .
$$

The representation $\mathcal{T}$ induced by $\mathcal{S}$ is then defined [40, 41] as the homomorphism $\mathcal{T}: H=G \ltimes_{\sigma} A \rightarrow \operatorname{GL}(\mathcal{H}),(f, \alpha) \mapsto \mathcal{T}[(f, \alpha)]$ with

$$
(\mathcal{T}[(f, \alpha)] \Psi)(q):=e^{i\langle q, \alpha\rangle} \mathcal{R}\left[g_{q}^{-1} f g_{f^{-1} \cdot q}\right] \Psi\left(f^{-1} \cdot q\right) \quad \forall q \in \mathcal{O}_{p}, \forall \Psi \in \mathcal{H}
$$

One easily verifies that this representation is unitary with respect to the scalar product (2.8). Note that $g_{q}^{-1} f g_{f^{-1} \cdot q}$ belongs to the little group $G_{p}$ for all $f \in G$ and any $q \in \mathcal{O}_{p}$.

As briefly recalled in the introduction, Mackey's main theorem [36-40] for separable, locally compact groups states that, provided the semi-direct product $H$ is regular, every unitary irreducible representation of $H$ is unitarily equivalent to an induced representation. 
Delta function basis. Definition (2.9) of the induced representation $\mathcal{T}$ can be written in an alternative form, that can sometimes be more convenient [41]. Consider the $G$-invariant delta function $\delta_{\mu}$ associated with the measure $\mu$ on $\mathcal{O}_{p}$,

$$
\int_{\mathcal{O}_{p}} d \mu(q) \delta_{\mu}(q-k) \varphi(q)=\varphi(k) \quad \forall \varphi, \forall k \in \mathcal{O}_{p}
$$

Then, if $\left\{e_{m} \mid m=1,2,3, \ldots\right\}$ is an orthonormal basis of $\mathcal{E}$, define the states

$$
\Psi_{k, m}(q):=\delta_{\mu}(q-k) e_{m},
$$

which form an (improper) orthonormal basis of $\mathcal{H}$ :

$$
\left\langle\Psi_{k, m} \mid \Psi_{\ell, n}\right\rangle=\delta_{m n} \delta_{\mu}(k-\ell)
$$

The induced representation (2.9) then acts on these states according to

$$
\mathcal{T}[(f, \alpha)] \Psi_{k, m}=e^{i\langle f \cdot k, \alpha\rangle}\left(\mathcal{R}\left[g_{f \cdot k}^{-1} f g_{k}\right]\right)_{m}^{n} \Psi_{f \cdot k, n} .
$$

Note that, assuming irreducibility of $\mathcal{R}$, the irreducibility of $\mathcal{T}$ is obvious in this formulation: since $\mathcal{R}$ is irreducible, and since the delta function basis is defined only on the orbit $\mathcal{O}_{p}$, there always exists a transformation $f$ that maps $\Psi_{k, m}$ onto $\Psi_{\ell, n}$, for all $k, \ell$ and any $m, n$.

\subsection{The case of the adjoint action}

A particular case, that will be relevant for $\mathrm{BMS}_{3}$, is the case where $G$ is a Lie group, $A=\mathfrak{g}_{\mathrm{ab}}$ is its Lie algebra (seen as the abelian additive group of a vector space) and $\sigma=\operatorname{Ad}$ is the adjoint action,

$$
H=G \ltimes \text { Ad } \mathfrak{g}_{\mathrm{ab}} .
$$

In this case, the orbits $\mathcal{O}_{p}$ defined in (2.4) coincide with the coadjoint orbits of $G$. The little groups $G_{p}$ are Lie groups and the associated Lie algebras, the little algebras, are denoted by $\mathfrak{g}_{p}$.

The Lie algebra of (2.13) is the semi-direct sum

$$
\mathfrak{h}=\mathfrak{g} \oplus_{\Sigma} \mathfrak{g}_{\mathrm{ab}},
$$

where $\mathfrak{g}_{\mathrm{ab}}$ denotes the abelian Lie algebra that is isomorphic to $\mathfrak{g}$ as a vector space. According to $(2.2)$, the Lie bracket in $\mathfrak{h}$ then reads

$$
[(X, \alpha),(Y, \beta)]=([X, Y],[X, \beta]-[Y, \alpha]) .
$$

The additional structure that is available in this setting is a Poisson bracket on $\mathfrak{g}^{*}$ [54]. In the finite-dimensional case, if $x_{a}$ are coordinates on $\mathfrak{g}^{*}$ associated with a dual basis $e_{*}^{a}$, the bracket is given by

$$
\{F, G\}=\frac{\partial F}{\partial x_{a}} C_{a b}^{c} \frac{\partial G}{\partial x_{b}} x_{c}, \quad F, G \in C^{\infty}\left(\mathfrak{g}^{*}\right),
$$

where $C_{b c}^{a}$ are the structure constants in the basis $e_{a}$ of $\mathfrak{g}$. If $G$ is connected, its coadjoint orbits are the leaves of the symplectic foliation induced by this bracket on $\mathfrak{g}^{*}$. The 
coadjoint action of a group element $f$ preserves these leaves and is generated by a suitable Hamiltonian. The $G$-invariant symplectic form $\omega$ on $\mathcal{O}_{p}$ is then defined by

$$
\omega_{q}\left(\operatorname{ad}_{X}^{*} q, \operatorname{ad}_{Y}^{*} q\right):=\langle q,[X, Y]\rangle \quad \forall q \in \mathcal{O}_{p}, \forall X, Y \in \mathfrak{g} .
$$

Note that the tangent space of $\mathcal{O}_{p}$ at $q$ consists of coadjoint vectors of the form $\operatorname{ad}_{X}^{*} q$. If the orbit $\mathcal{O}_{p}$ is $2 n$-dimensional, the $2 n$-form $\omega^{n} / n$ ! is a $G$-invariant volume form on $\mathcal{O}_{p}$; when considering induced representations of $G \ltimes_{\text {Ad }} \mathfrak{g}_{\mathrm{ab}}$, it can be used to define the scalar product (2.8).

\subsection{Remarks on projective representations}

In applications to quantum mechanics, one is primarily interested in projective unitary representations, i.e. unitary representations up to a phase [66]. Bargmann's results [64] state that each projective representation of a group $G$ can be identified with an ordinary representation of the central extension $\widehat{G}$ of $G$. The latter is determined by the second cohomology group $H^{2}(G, \mathbb{R})$.

One can show that for a connected Lie group $G$ with vanishing Lie algebra cohomology in degree two, projective representations are standard representations multiplied by phase factors forming a representation of the fundamental group $\pi_{1}(G)$ of $G$. If in addition $G$ is simply connected, then $H^{2}(G, \mathbb{R})=0$ and every projective unitary representation of $G$ can be lifted to an ordinary unitary representation.

This is the reason why, in discussing representations, one considers the universal covering $\mathrm{SL}(2, \mathbb{C}) \ltimes L^{2}\left(S^{2}\right)$ of the globally well-defined $\mathrm{BMS}_{4}$ group, and also the centrally extended Virasoro group $\widehat{\operatorname{Diff}^{+}}\left(S^{1}\right)$ and its universal cover.

\section{The Poincaré group in three dimensions}

In this section, we briefly recall results on representations of the Poincaré group in three dimensions, the exact isometry group of three-dimensional Minkowski spacetime, in order to compare them with those of the $\mathrm{BMS}_{3}$ group. This is motivated by the fact that (i) the Poincaré group is a subgroup of $\mathrm{BMS}_{3}$, (ii) it satisfies all the assumptions required by Mackey's theorem on induced representations, and (iii) it is a simple example of a semi-direct product of the form $G \ltimes_{\mathrm{Ad}} \mathfrak{g}_{\mathrm{ab}}$.

\subsection{Double cover of the Poincaré group}

The Poincaré group in three spacetime dimensions is the semi-direct product of the connected Lorentz group SO $(2,1)^{\uparrow}$ with the abelian vector group $\mathbb{R}^{3}$ of spacetime translations:

$$
P_{3}^{\uparrow}:=\mathrm{SO}(2,1)^{\uparrow} \ltimes_{\sigma} \mathbb{R}^{3},
$$

where $\sigma$ is the vector representation of $\mathrm{SO}(2,1)$. Explicitly, if elements of the Poincaré group are written as pairs $(\Lambda, a)$, where $\Lambda \in \mathrm{SO}(2,1)^{\uparrow}$ and $a=\left(a^{0}, a^{1}, a^{2}\right) \in \mathbb{R}^{3}$, then Lorentz transformations act on translations according to $\left(\sigma_{\Lambda} a\right)^{\mu}=\Lambda_{\nu}^{\mu}{ }_{\nu} a^{\nu}$ with $\mu, \nu, \ldots=0,1,2$. 
When one is interested in projective representations, it is useful to consider, instead of $P_{3}^{\uparrow}$, its double cover

$$
\bar{P}_{3}^{\uparrow}:=\operatorname{SL}(2, \mathbb{R}) \ltimes_{\mathrm{Ad}} \mathfrak{s l}(2, \mathbb{R})_{\mathrm{ab}},
$$

which follows from the standard isomorphism $\operatorname{SO}(2,1)^{\uparrow} \cong \operatorname{SL}(2, \mathbb{R}) / \mathbb{Z}_{2}:=\operatorname{PSL}(2, \mathbb{R})$. To show this isomorphism, one begins by identifying $\mathbb{R}^{3}$ with $\mathfrak{s l}(2, \mathbb{R})_{\mathrm{ab}}$, associating with a translation vector $a$ a traceless $2 \times 2$ matrix $\alpha:=a^{\mu} t_{\mu}$, where the generators $t_{\mu}$ of $\mathfrak{s l}(2, \mathbb{R})$ can be chosen as

$$
t_{0}=\frac{1}{2}\left(\begin{array}{cc}
0 & 1 \\
-1 & 0
\end{array}\right), \quad t_{1}=\frac{1}{2}\left(\begin{array}{ll}
0 & 1 \\
1 & 0
\end{array}\right), \quad t_{2}=\frac{1}{2}\left(\begin{array}{cc}
1 & 0 \\
0 & -1
\end{array}\right) .
$$

They satisfy $t_{\mu} t_{\nu}=\frac{1}{2} \epsilon_{\mu \nu \rho} t^{\rho}+\frac{1}{4} \eta_{\mu \nu} \mathbb{I}$, where $\eta_{\mu \nu}:=\operatorname{diag}(-1,1,1)$ and $\epsilon_{012}:=1$; as usual, $\eta_{\mu \nu}$ and its inverse will be used below to lower and raise indices. The invariant bilinear form on $\mathfrak{s l}(2, \mathbb{R})$ reproduces the Minkowskian scalar product of vectors:

$$
\langle\alpha, \beta\rangle=2 \operatorname{Tr}(\alpha \beta)=\eta_{\mu \nu} a^{\mu} b^{\nu} .
$$

The isomorphism then follows from the surjective homomorphism $H: \operatorname{SL}(2, \mathbb{R}) \rightarrow$ SO $(2,1)^{\uparrow}, f \mapsto H[f]$ such that

$$
f t_{\mu} f^{-1} a^{\mu}=t_{\nu}(H[f])_{\rho}^{\nu} a^{\rho} \quad \forall a^{\mu} t_{\mu} \in \mathfrak{s l}(2, \mathbb{R}),
$$

whose kernel is isomorphic to $\mathbb{Z}_{2}$. For later use, we note that if

$$
f=\left(\begin{array}{ll}
a & b \\
c & d
\end{array}\right) \in \mathrm{SL}(2, \mathbb{R})
$$

then

$$
H[f]=\left(\begin{array}{ccc}
\frac{1}{2}\left(a^{2}+b^{2}+c^{2}+d^{2}\right) & \frac{1}{2}\left(a^{2}-b^{2}+c^{2}-d^{2}\right) & -a b-c d \\
\frac{1}{2}\left(a^{2}+b^{2}-c^{2}-d^{2}\right) & \frac{1}{2}\left(a^{2}-b^{2}-c^{2}+d^{2}\right) & -a b+c d \\
-a c-b d & b d-a c & a d+b c
\end{array}\right) .
$$

From now on, when talking about the Poincaré group in three dimensions, we will refer to its double cover (3.1).

It follows from the structure $G \ltimes$ Ad $\mathfrak{g}_{\mathrm{ab}}$ of the Poincaré group that its Lie algebra has the general form (2.14). In terms of the basis

$$
j_{-1}:=-i\left(t_{0}+t_{1}\right), \quad j_{1}:=-i\left(t_{0}-t_{1}\right), \quad j_{0}:=-i t_{2}
$$

of $\mathfrak{s l}(2, \mathbb{R})$ and of its counterpart $\left\{p_{-1}, p_{1}, p_{0}\right\}$ in $\mathfrak{s l}(2, \mathbb{R})_{\text {ab }}$, the commutation relations of the three-dimensional Poincaré algebra are

$$
i\left[j_{m}, j_{n}\right]=(m-n) j_{m+n}, \quad i\left[j_{m}, p_{n}\right]=(m-n) p_{m+n}, \quad i\left[p_{m}, p_{n}\right]=0,
$$

with $m, n=-1,0,1$. The $j_{m}$ 's are thus to be interpreted as Lorentz generators, while the $p_{m}$ 's are generators of translations.

Note that $\mathrm{SL}(2, \mathbb{R})$ is not simply connected: its fundamental group is isomorphic to $\mathbb{Z}$, as is that of the Poincaré group in three dimensions. The latter thus admits genuinely projective unitary representations, whose classification relies on its universal cover. 


\subsection{Induced representations of the Poincaré group}

Coadjoint orbits of $\mathbf{S L}(\mathbf{2}, \mathbb{R})$. The existence of the invariant bilinear form (3.3) implies that adjoint and coadjoint representations of $\mathrm{SL}(2, \mathbb{R})$ are equivalent, so that coadjoint orbits can be deduced from adjoint ones. If $\left\{t^{0 *}, t^{1 *}, t^{2 *}\right\}$ denotes the dual basis of $\mathfrak{s l}(2, \mathbb{R})^{*}$ associated with $(3.2)$, write $p=p_{\mu} t^{\mu *} \in \mathfrak{s l}(2, \mathbb{R})^{*}$. The equivalence follows by associating, with each such coadjoint vector $p$, the translation vector $p^{\mu} t_{\mu} \in \mathfrak{s l}(2, \mathbb{R})$.

Fix an arbitrary number $\kappa>0$. It can then be shown that each coadjoint orbit $\mathcal{O}_{p}$ of $\mathrm{SL}(2, \mathbb{R})$ is of one of the following six types:

1. Upper hyperboloid $q_{\mu} q^{\mu}=-m^{2}, q_{0}>0, m>0$. Orbit representative $p=m t^{0 *}$. Little group $\mathrm{U}(1)$.

2. Lower hyperboloid $q_{\mu} q^{\mu}=-m^{2}, q_{0}<0, m>0$. Orbit representative $p=-m t^{0 *}$. Little group $\mathrm{U}(1)$.

3. Upper null cone $q_{\mu} q^{\mu}=0, q_{0}>0$. Orbit representative $p=\kappa\left(t^{0 *}+t^{1 *}\right)$. Little group $\mathbb{R} \times \mathbb{Z}_{2}$.

4. Lower null cone $q_{\mu} q^{\mu}=0, q_{0}<0$. Orbit representative $p=-\kappa\left(t^{0 *}+t^{1 *}\right)$. Little group $\mathbb{R} \times \mathbb{Z}_{2}$.

5. One-sheeted hyperboloid $q_{\mu} q^{\mu}=m^{2}$. Orbit representative $p=m t^{2 *}$. Little group $\mathbb{R} \times \mathbb{Z}_{2}$.

6. Trivial orbit $p=0$. Little group $\operatorname{SL}(2, \mathbb{R})$.

Energy-momentum. When interpreting unitary irreducible representations of the Poincaré group as one-particle states of a relativistic particle, $p$ is its energy-momentum vector. Particles on the lower/upper hyperboloids are massive, the sign of $p_{0}$ determining whether they propagate towards the future or the past, while particles on the one-sheeted hyperboloids move faster than light — they are tachyons.

Spin. In building induced representations, one also needs unitary irreducible representations of the little groups. In the massive case with U(1) little groups, these representations are one-dimensional, and are labelled by an integer $j$. When considering projective representations, the universal cover of the Poincaré group becomes relevant and these U(1)'s are replaced by $\mathbb{R}$. The label $j$ becomes an arbitrary real number and there is no quantization of spin.

In the massless case, the unitary irreducible representations of the little groups $\mathbb{R} \times \mathbb{Z}_{2}$ are characterized by spins, also called helicities, $s \in \mathbb{R}$ and $\epsilon= \pm 1$.

Finally, the trivial orbit has little group $\mathrm{SL}(2, \mathbb{R})$, which admits three series of infinitedimensional unitary irreducible representations. The trivial representation corresponds to the vacuum state. 
Interpretation of induced representations. The transformations $g_{q}$ introduced in (2.6), and defined so that $g_{q} \cdot p=q$, correspond here to standard boosts that map an orbit representative $p$ (e.g. the momentum of a massive particle at rest) on a momentum $q$ belonging to its orbit (representing the momentum of the same particle with non-zero velocity). The composite Lorentz transformation $g_{q}^{-1} f g_{f^{-1} \cdot q}$ appearing in (2.9) is then called a Wigner rotation.

For the orbits $\mathcal{O}_{p}$ of future-directed massive particles in three dimensions, the Lorentzinvariant measure appearing in the scalar product (2.8) is given by the volume form

$$
d \mu(q)=\frac{d q_{1} d q_{2}}{2 q_{0}}, \quad q_{0}=\sqrt{m^{2}+q_{1}^{2}+q_{2}^{2}}
$$

This form coincides (up to normalization) with the symplectic form (2.16) on $\mathcal{O}_{p}$.

For all orbits except the trivial one, the little group is one-dimensional and the corresponding space $\mathcal{E}$ introduced above (2.7) is just $\mathbb{C}$, so that the Hilbert space $\mathcal{H}$ is the space of complex-valued quantum wavefunctions in momentum space. The induced representation (2.9) acts by simultaneously boosting, rotating and translating these wavefunctions. Each element $\Psi_{k}$ of the delta function basis (2.10) then represents a plane wave of definite momentum $k$ and spin $j$.

\section{Virasoro algebra and group}

Here we recall some basic definitions regarding the Virasoro group, expressed in terms of functions on the circle. We refer for instance to [69] for a more detailed review.

\subsection{Virasoro algebra}

Vector fields on the circle. Smooth functions on $S^{1}$ can be identified with $2 \pi$-periodic smooth functions of a variable $\phi \in \mathbb{R}$. Let $\operatorname{Vect}\left(S^{1}\right)$ denote the Lie algebra of vector fields on the circle. Its elements are written as $X=X(\phi) \frac{\partial}{\partial \phi}$, where $X(\phi)$ is a smooth function, the Lie bracket being $[X, Y]=\left(X Y^{\prime}-Y X^{\prime}\right) \frac{\partial}{\partial \phi}$. In terms of the Fourier basis $\ell_{m}:=i e^{i m \phi} \frac{\partial}{\partial \phi}$, the commutation relations of $\operatorname{Vect}\left(S^{1}\right) \operatorname{read}$

$$
\left[\ell_{m}, \ell_{n}\right]=(m-n) \ell_{m+n}
$$

Tensor densities on the circle. The space of tensor densities $\mathcal{F}_{\lambda}$ of degree $\lambda \in \mathbb{R}$ consists of elements of the form $\alpha=\alpha(\phi) d \phi^{\lambda}$, with $\alpha(\phi)$ a smooth function. It is a $\operatorname{Vect}\left(S^{1}\right)$-module with action

$$
\Sigma_{X}^{\lambda} \alpha=X \alpha^{\prime}+\lambda X^{\prime} \alpha
$$

In particular, $\mathcal{F}_{-1} \cong \operatorname{Vect}\left(S^{1}\right)$ as a $\operatorname{Vect}\left(S^{1}\right)$-module, and the action of $\operatorname{Vect}\left(S^{1}\right)$ on $\mathcal{F}_{-1}$ coincides with its adjoint action.

Note that the bilinear form on $\mathcal{F}_{\lambda} \times \mathcal{F}_{1-\lambda}$ given by

$$
\langle\alpha, \beta\rangle:=\int_{S^{1}} \alpha \otimes \beta \quad \text { for } \alpha \in \mathcal{F}_{\lambda}, \beta \in \mathcal{F}_{1-\lambda}
$$


is invariant under the action of $\operatorname{Vect}\left(S^{1}\right)$ in the sense that $\left\langle\Sigma_{X}^{\lambda} \alpha, \beta\right\rangle+\left\langle\alpha, \Sigma_{X}^{1-\lambda} \beta\right\rangle=0$. This bilinear form can be used to identify the regular dual $\operatorname{Vect}\left(S^{1}\right)^{*}$ with the space $\mathcal{F}_{2}$ of quadratic densities: for $p=p(\phi) d \phi^{2} \in \mathcal{F}_{2}$,

$$
\langle p, \alpha\rangle=\int_{0}^{2 \pi} p(\phi) \alpha(\phi) d \phi \quad \forall \alpha \in \mathcal{F}_{-1}
$$

The action of $\operatorname{Vect}\left(S^{1}\right)$ on $\mathcal{F}_{2}$ then coincides with its coadjoint action: $\Sigma_{X}^{2} p=\operatorname{ad}_{X}^{*} p=$ $\left(X p^{\prime}+2 X^{\prime} p\right) d \phi^{2}$.

Centrally extended algebra. The cohomology $\operatorname{ring} H^{*}\left(\operatorname{Vect}\left(S^{1}\right), \mathbb{R}\right)$ is generated by elements in degrees $0,2,3$, where the cohomology group is one-dimensional. In particular, a representative in degree 2 is given by the Gelfand-Fuchs cocycle

$$
C(X, Y)=\int_{0}^{2 \pi} d \phi \operatorname{det}\left(\begin{array}{ll}
X^{\prime} & Y^{\prime} \\
X^{\prime \prime} & Y^{\prime \prime}
\end{array}\right)
$$

whose expression in the Fourier basis is $C\left(\ell_{m}, \ell_{n}\right)=4 \pi i m^{3} \delta_{m+n}^{0}$. The second cohomology group $H^{2}\left(\operatorname{Vect}\left(S^{1}\right), \mathbb{R}\right)$ is directly related to $H^{1}\left(\operatorname{Vect}\left(S^{1}\right), \mathcal{F}_{2}\right)$, which is also onedimensional, with representative $s[X]=2 X^{\prime \prime \prime} d \phi^{2}$.

The Virasoro algebra $\widehat{\operatorname{Vect}}\left(S^{1}\right)$ is the universal central extension of $\operatorname{Vect}\left(S^{1}\right)$ and consists of pairs $(X,-i a)$, where $a \in \mathbb{R}$. The associated Lie bracket reads

$$
[(X,-i a),(Y,-i b)]=\left([X, Y],-\frac{i}{48 \pi} C(X, Y)\right) .
$$

Equivalently, in terms of the Fourier basis $L_{m}:=\left(\ell_{m}, \frac{1}{24} \delta_{m}^{0}\right), Z:=(0,1)$, the commutation relations of the Virasoro algebra read

$$
\left[L_{m}, L_{n}\right]=(m-n) L_{m+n}+\frac{1}{12} \delta_{m+n}^{0} m\left(m^{2}-1\right) Z, \quad\left[Z, L_{m}\right]=0
$$

The dual space $\widehat{\operatorname{Vect}}\left(S^{1}\right)^{*}$ of the Virasoro algebra consists of pairs $(p, i c)$ with $c \in \mathbb{R}$, paired with $\widehat{\operatorname{Vect}}\left(S^{1}\right)$ according to

$$
\langle(p, i c),(X,-i a)\rangle=\int_{0}^{2 \pi} d \phi p X+c a
$$

The coadjoint representation of the Virasoro algebra then reads

$$
\operatorname{ad}_{X}^{*}(p, i c)=\left(\left[X p^{\prime}+2 X^{\prime} p-\frac{c}{24 \pi} X^{\prime \prime \prime}\right] d \phi^{2}, 0\right) .
$$

Here and below, we drop the central element -ia in the subscript of the coadjoint action, since it acts trivially. 


\subsection{Virasoro group}

Diffeomorphisms of the circle. The group of orientation-preserving diffeomorphisms of the circle will be denoted by $\operatorname{Diff}^{+}\left(S^{1}\right)$. It can be endowed with the structure of a Lie-Fréchet group with Lie algebra $\operatorname{Vect}\left(S^{1}\right)$. Similar notations and properties hold when replacing $S^{1}$ with $\mathbb{R}$. Diff ${ }^{+}\left(S^{1}\right)$ is connected, but not simply connected: its fundamental group is $\pi_{1}\left(\operatorname{Diff}^{+}\left(S^{1}\right)\right) \cong \mathbb{Z}$. Its universal cover $\widetilde{\operatorname{Diff}^{+}}\left(S^{1}\right)$, whose elements will be denoted as $f, g$, etc., is the subgroup of $\operatorname{Diff}^{+}(\mathbb{R})$ consisting of $2 \pi \mathbb{Z}$-equivariant diffeomorphisms

$$
f: \mathbb{R} \rightarrow \mathbb{R}, \phi \mapsto f(\phi) \quad \text { such that } f^{\prime}(\phi)>0 \text { and } f(\phi+2 \pi)=f(\phi)+2 \pi .
$$

The corresponding projection $\chi: \widetilde{\operatorname{Diff}^{+}}\left(S^{1}\right) \rightarrow \operatorname{Diff}^{+}\left(S^{1}\right), f \mapsto F$ is defined through

$$
e^{i f(\phi)}=F\left(e^{i \phi}\right),
$$

so that

$$
\operatorname{Diff}^{+}\left(S^{1}\right)=\widetilde{\operatorname{Diff}^{+}}\left(S^{1}\right) / 2 \pi \mathbb{Z}
$$

where $2 \pi \mathbb{Z}$ is identified with the subgroup of $\widetilde{\operatorname{Diff}^{+}}\left(S^{1}\right)$ consisting of translations of $\mathbb{R}$ by integer multiples of $2 \pi$.

For future use, it is convenient to associate with $f \in \widetilde{\operatorname{Diff}^{+}}\left(S^{1}\right)$ the function

$$
\theta(\phi):=\frac{1}{\left(f^{-1}\right)^{\prime}(\phi)},
$$

which is positive, $2 \pi$-periodic, and satisfies

$$
\int_{0}^{2 \pi} \frac{d \phi}{\theta(\phi)}=2 \pi
$$

Tensor densities on the circle. $\widetilde{\operatorname{Diff}}^{+}\left(S^{1}\right)$ acts on $\mathcal{F}_{\lambda}$ according to

$$
\sigma_{f^{-1}}^{\lambda}(\alpha):=\left(f^{\prime}\right)^{\lambda} \alpha \circ f
$$

For $\lambda=-1$, this action coincides with the adjoint action when taking into account that $\mathcal{F}_{-1} \cong \operatorname{Vect}\left(S^{1}\right)$. The bilinear form defined in (4.2) is invariant in the sense that $\left\langle\sigma_{f}^{\lambda} \alpha, \sigma_{f}^{1-\lambda} \beta\right\rangle=\langle\alpha, \beta\rangle$.

Centrally extended group. The second cohomology group $H^{2}\left(\widetilde{\operatorname{Diff}^{+}}\left(S^{1}\right), \mathbb{R}\right)$ is onedimensional and can be represented by the Bott-Thurston cocycle

$$
B(f, g)=\int_{0}^{2 \pi} d \phi \ln \left(f^{\prime} \circ g\right)\left(\ln \left(g^{\prime}\right)\right)^{\prime} .
$$

For simplicity, we define ${ }^{1}$ the Virasoro group $\widehat{\operatorname{Diff}^{+}}\left(S^{1}\right)=\widetilde{\operatorname{Diff}^{+}}\left(S^{1}\right) \times \mathbb{R}$ to be the central extension of $\widetilde{\operatorname{Diff}^{+}}\left(S^{1}\right)$. It consists of pairs $(f,-i a)$, the group law being

$$
(f,-i a) \cdot(g,-i b)=\left(f \circ g,-i\left(a+b-\frac{1}{48 \pi} B(f, g)\right)\right) .
$$

\footnotetext{
${ }^{1}$ There is an alternative definition of both the Virasoro and the $\mathrm{BMS}_{3}$ groups that involves Diff ${ }^{+}\left(S^{1}\right)$ instead of its universal cover. What we call the Virasoro and $\mathrm{BMS}_{3}$ groups here corresponds to the universal cover of these groups in the alternative description.
} 
The Lie algebra of the Virasoro group is the Virasoro algebra (4.4), up to an overall sign in the commutation relations.

As in the case of $\operatorname{Vect}\left(S^{1}\right)$, there is a direct relation between $H^{2}\left(\widetilde{\operatorname{Diff}^{+}}\left(S^{1}\right), \mathbb{R}\right)$ and $H^{1}\left(\widetilde{\operatorname{Diff}^{+}}\left(S^{1}\right), \mathcal{F}_{2}\right)$. The cocycle associated in this way with $B(f, g)$ is $2 S[f] d \phi^{2}$, where

$$
S[f]:=\frac{f^{\prime \prime \prime}}{f^{\prime}}-\frac{3}{2}\left(\frac{f^{\prime \prime}}{f^{\prime}}\right)^{2}
$$

is the Schwarzian derivative of $f$. It is the finite form of the infinitesimal cocycle $s$ introduced below (4.3). The cocycle condition is then equivalent to the identity

$$
S[f \circ g]=(S[f] \circ g)\left(g^{\prime}\right)^{2}+S[g] .
$$

Note that $S[h](x)=0$ iff $h(x)=(a x+b) /(c x+d)$, with $a d-b c=1$. When the coordinate $x$ describes the projective line, such transformations form a group isomorphic to $\operatorname{PSL}(2, \mathbb{R})$; however, when $x=\phi$ is a coordinate on the circle, the only transformation of this form that is also a diffeomorphism of the circle is $h(\phi)=\phi+$ cst.

Consider the function $t_{n}(\phi)=\tan (n \phi / 2)$ related to the stereographic projection of the $n$-fold cover of the circle. From $S\left[t_{n}\right]=n^{2} / 2$ and eq. (4.13), it follows that $S\left[t_{n} \circ f \circ t_{n}^{-1}\right]=$ $\left(\bar{S}_{n}[f] \circ t_{n}^{-1}\right)\left(\left(t_{n}^{-1}\right)^{\prime}\right)^{2}$, with the definition

$$
\bar{S}_{n}[f]:=S[f]+\frac{n^{2}}{2}\left(\left(f^{\prime}\right)^{2}-1\right) .
$$

An important property of the Schwarzian derivative is the inequality

$$
\int_{0}^{2 \pi} d \phi \bar{S}_{1}[f] \leqslant 0 \quad \forall f \in \widetilde{\operatorname{Diff}^{+}}\left(S^{1}\right)
$$

or, equivalently, $\int_{0}^{2 \pi} d \phi\left[\theta-\left(\theta^{\prime}\right)^{2} / \theta\right] \leqslant 2 \pi$ in terms of the $\theta$ function in (4.9). This inequality is saturated iff $f$ is given by

$$
e^{i f(\phi)}=\frac{\alpha e^{i \phi}+\beta}{\bar{\beta} e^{i \phi}+\bar{\alpha}}, \quad|\alpha|^{2}-|\beta|^{2}=1,
$$

that is, iff $f$ is the lift of a projective transformation of the circle.

Finally, the coadjoint action of the Virasoro group is given by

$$
\operatorname{Ad}_{f^{-1}}^{*}(p, i c)=\left(\left[\left(f^{\prime}\right)^{2} p \circ f-\frac{c}{24 \pi} S[f]\right] d \phi^{2}, i c\right) .
$$

The associated differential is (4.6), up to an overall minus sign.

\section{BMS group in three dimensions}

We now provide the abstract definition of $\mathrm{BMS}_{3}$ in terms of functions on the circle, before passing to its more standard description on null infinity and isolating its Poincaré subgroups. 


\subsection{Structure of the group and algebra}

The (centerless) $\mathrm{BMS}_{3}$ group is the symmetry group of three-dimensional asymptotically flat spacetimes at (future or past) null infinity. It is the semi-direct product of superrotations and supertranslations under the adjoint action:

$$
\mathrm{BMS}_{3}:=\widetilde{\operatorname{Diff}^{+}}\left(S^{1}\right) \ltimes_{\mathrm{Ad}} \operatorname{Vect}\left(S^{1}\right)_{\mathrm{ab}} .
$$

The group of supertranslations is the abelian additive group $\operatorname{Vect}\left(S^{1}\right)_{\mathrm{ab}}$, while the group of superrotations ${ }^{2}$ is $\widetilde{\operatorname{Diff}^{+}}\left(S^{1}\right)$. Elements of $\mathrm{BMS}_{3}$ are denoted by $(f, \alpha)$, the adjoint action being (4.11) with $\lambda=-1$. The Lie algebra of $\mathrm{BMS}_{3}$ is the semi-direct sum

$$
\mathfrak{b m s}_{3}=\operatorname{Vect}\left(S^{1}\right) \oplus_{\mathrm{ad}} \operatorname{Vect}\left(S^{1}\right)_{\mathrm{ab}} .
$$

Introducing a Fourier basis of the latter by defining

$$
j_{m}:=\left(e^{i m \phi} \frac{\partial}{\partial \phi}, 0\right) \quad \text { and } \quad p_{m}:=\left(0, e^{i m \phi} d \phi^{-1}\right),
$$

the commutation relations of $\mathfrak{b m s}_{3}$ are of the form (3.6), except that now the indices $m, n$ run over all integer values. This makes explicit the fact that the $\mathfrak{b m s}_{3}$ algebra is an infinite-dimensional extension of the Poincaré algebra in three dimensions.

As regards projective representations, the centrally extended $\mathrm{BMS}_{3}$ group is relevant:

$$
\widehat{\mathrm{BMS}}_{3}=\widehat{\operatorname{Diff}^{+}}\left(S^{1}\right) \ltimes_{\mathrm{Ad}} \widehat{\operatorname{Vect}}\left(S^{1}\right)_{\mathrm{ab}} .
$$

Its elements are quadruples $(f,-i a ; \alpha,-i b)$ and the adjoint action reads

$$
\operatorname{Ad}_{f}(\alpha,-i b)=\left(\left(\alpha f^{\prime}\right) \circ f^{-1}, \frac{i}{24 \pi} \int_{0}^{2 \pi} d \phi S[f] \alpha-i b\right) .
$$

Up to an overall minus sign in the Lie brackets, the associated Lie algebra is

$$
\widehat{\mathfrak{b m s}_{3}}=\widehat{\operatorname{Vect}}\left(S^{1}\right) \oplus_{\mathrm{ad}} \widehat{\operatorname{Vect}}\left(S^{1}\right)_{\mathrm{ab}},
$$

whose elements are quadruples $(X,-i a ; \alpha,-i b)$ with commutation relations

$$
\begin{aligned}
& {[(X,-i a ; \alpha,-i b),(Y,-i r ; \beta,-i s)]=} \\
& \quad=\left([X, Y],-\frac{i}{48 \pi} C(X, Y) ;[X, \beta]-[Y, \alpha],-\frac{i}{48 \pi}(C(X, \beta)-C(Y, \alpha))\right) .
\end{aligned}
$$

In terms of the Fourier basis

$$
\begin{aligned}
J_{m}:=\left(e^{i m \phi} \frac{\partial}{\partial \phi}, \frac{-i}{24} \delta_{m}^{0} ; 0,0\right), & Z_{1}:=(0,1 ; 0,0), \\
P_{m}:=\left(0,0 ; e^{i m \phi} d \phi^{-1}, \frac{-i}{24} \delta_{m}^{0}\right), & Z_{2}:=(0,0 ; 0,1),
\end{aligned}
$$

the non-vanishing brackets of $\widehat{\mathfrak{b m s}}_{3}$ are

$$
\begin{aligned}
& i\left[J_{m}, J_{n}\right]=(m-n) J_{m+n}+\frac{Z_{1}}{12} m\left(m^{2}-1\right) \delta_{m+n}^{0}, \\
& i\left[J_{m}, P_{n}\right]=(m-n) P_{m+n}+\frac{Z_{2}}{12} m\left(m^{2}-1\right) \delta_{m+n}^{0} .
\end{aligned}
$$

\footnotetext{
${ }^{2}$ The reason for using the universal cover of the diffeomorphism group is that we are ultimately interested in projective representations, so we want our group of interest to be simply connected.
} 


\section{2 $\quad \mathrm{BMS}_{3}$ as transformation group on $\mathscr{I}$}

So far, $\mathrm{BMS}_{3}$ has been given a purely one-dimensional description. In the study of threedimensional asymptotically flat spacetimes, an equivalent two-dimensional description, in terms of transformations of (future or past) null infinity $\mathscr{I}=S^{1} \times \mathbb{R}$, appears naturally. Introducing local coordinates $(\phi, u)$ on $\mathscr{I}$, the transformation associated with $(f, \alpha) \in$ $\mathrm{BMS}_{3}$ is given by

$$
(\phi, u) \mapsto\left(f(\phi), f^{\prime}(\phi)(u+\alpha(\phi))\right)
$$

\subsection{Poincaré subgroups of $\mathrm{BMS}_{3}$}

In order to isolate the natural Poincaré subgroup of $\mathrm{BMS}_{3}$, it suffices to compute the action of the Poincaré group on $\mathscr{I}$. An easy way to do this on $\mathscr{I}^{+}$is to use BMS coordinates $r, u, \phi$ related to the standard cartesian coordinates $x^{\mu}$ of Minkowski spacetime through $r e^{i \phi}:=x^{1}+i x^{2}, u:=x^{0}-r$. Then $r \in \mathbb{R}^{+}$is a radial coordinate, $u \in \mathbb{R}$ is an outgoing null coordinate, and, as before, $\phi$ is an angular coordinate on $S^{1}$. In terms of BMS coordinates, the Minkowski metric reads

$$
d \bar{s}^{2}=-d u^{2}-2 d u d r+r^{2} d \phi^{2}
$$

Poincaré transformations $x^{\mu} \mapsto \Lambda_{\nu}^{\mu} x^{\nu}+a^{\mu}$ can then be expressed in BMS coordinates, and the limit $r \rightarrow+\infty$ yields their action on $\mathscr{I}^{+}$.

For a translation $x^{\mu} \mapsto x^{\mu}+a^{\mu}$, the limit $r \rightarrow+\infty$ simply produces

$$
(\phi, u) \mapsto(\phi, u+\alpha), \quad \alpha(\phi)=a^{0}-a^{1} \cos \phi-a^{2} \sin \phi
$$

For a Lorentz transformation, it is useful to refer to the homomorphism $H$ in (3.5), parametrizing Lorentz transformations by $\operatorname{SL}(2, \mathbb{R})$ matrices. Defining the complex variables

$$
\alpha:=\frac{1}{2}(a+d+i(b-c)), \quad \beta:=\frac{1}{2}(a-d-i(b+c)) \quad \text { such that }|\alpha|^{2}-|\beta|^{2}=1,
$$

a Lorentz transformation is realized on $\mathscr{I}^{+}$through the transformation $(\phi, u) \mapsto(\widetilde{\phi}, \widetilde{u})$, where

$$
e^{i \tilde{\phi}}=\frac{\alpha e^{i \phi}+\beta}{\bar{\beta} e^{i \phi}+\bar{\alpha}}, \quad\left(\begin{array}{cc}
\alpha & \beta \\
\bar{\beta} & \bar{\alpha}
\end{array}\right) \in \mathrm{SU}(1,1),
$$

and

$$
\widetilde{u}=\frac{u}{\left(\alpha e^{i \phi}+\beta\right)\left(\bar{\alpha} e^{-i \phi}+\bar{\beta}\right)}=\tilde{\phi}^{\prime} u .
$$

One verifies that this action correctly reproduces the SL $(2, \mathbb{R})$ group law. Note that the explicit form of the homomorphism (3.5) in terms of complex variables is

$$
H\left[\left(\begin{array}{ll}
a & b \\
c & d
\end{array}\right)\right]=\left(\begin{array}{ccc}
\alpha \bar{\alpha}+\beta \bar{\beta} & \alpha \bar{\beta}+\bar{\alpha} \beta & i(\alpha \bar{\beta}-\bar{\alpha} \beta) \\
\alpha \beta+\overline{\alpha \beta} & \frac{1}{2}\left(\alpha^{2}+\beta^{2}+\bar{\alpha}^{2}+\bar{\beta}^{2}\right) & \frac{i}{2}\left(\alpha^{2}-\beta^{2}-\bar{\alpha}^{2}+\bar{\beta}^{2}\right) \\
i(\overline{\alpha \beta}-\alpha \beta) & \frac{i}{2}\left(\bar{\alpha}^{2}+\bar{\beta}^{2}-\alpha^{2}-\beta^{2}\right) & \frac{1}{2}\left(\alpha^{2}-\beta^{2}+\bar{\alpha}^{2}-\bar{\beta}^{2}\right)
\end{array}\right)
$$


The natural Poincaré subgroup of $\mathrm{BMS}_{3}$ thus consists of pairs $(f, \alpha)$ whose $f$ is of the form $f(\phi)=\tilde{\phi}(\phi)$ given by (5.10), and whose $\alpha$ is a translation of the form (5.8). This embedding also justifies the terminology of "superrotations" and "supertranslations" introduced above.

Observe that $\mathrm{BMS}_{3}$ actually contains infinitely many distinct Poincaré subgroups obtained by taking the $n$-fold covers of $S^{1}$ (with $n \in \mathbb{N}^{*}$ ). These subgroups act on the cylinder $(\phi, u)$ at null infinity through the transformations obtained upon replacing $\tilde{\phi}$ and $\phi$ by $n \tilde{\phi}$ and $n \phi$ in equations (5.8), (5.10) and (5.11). The associated Poincaré subalgebras of $\mathfrak{b m s}_{3}$ are generated by the elements $p_{0}, p_{n}, p_{-n}, j_{0}, j_{n}$ and $j_{-n}$ of the Fourier basis (5.3).

\section{Induced representations of $\mathrm{BMS}_{3}$}

This section is devoted to the discussion of $\mathrm{BMS}_{3}$ particles, that is, unitary irreducible representations of $\widehat{\mathrm{BMS}}_{3}$ obtained by the inducing construction. As follows from the structure of this group, the orbits that are involved are the coadjoint orbits of the Virasoro group. We therefore revisit their classification and comment on their physical meaning in the present context by taking inspiration from the Poincaré group.

\subsection{Little algebras}

In the $\mathrm{BMS}_{3}$ context, elements of $\widehat{\operatorname{Vect}}\left(S^{1}\right)_{\mathrm{ab}}^{*}$ are pairs $\left(p, i c_{2}\right)$, where $c_{2}$ is the central charge related to $Z_{2}$ in (5.7). The little algebra $\mathfrak{g}_{p, c_{2}}$ then consists of vector fields $X \in \operatorname{Vect}\left(S^{1}\right)$ such that $\operatorname{ad}_{X}^{*}\left(p, i c_{2}\right)=0$, or explicitly, using eq. (4.6),

$$
X p^{\prime}+2 X^{\prime} p-\frac{c_{2}}{24 \pi} X^{\prime \prime \prime}=0 .
$$

The solutions $X$ of this equation depend on the central charge $c_{2}$ and on the form of $p(\phi)$.

- For vanishing central charge $c_{2}$, denote by $Z$ the set of zeros of $p(\phi)$; there are three families of solutions:

1. If $Z=\varnothing$, the little algebra is one-dimensional.

2. If $Z \neq \varnothing$ and int $Z=\varnothing$, the little algebra is trivial.

3. If $Z \neq \varnothing$ and int $Z \neq \varnothing$, the little algebra consists of vector fields whose support is contained in $Z$.

- For non-zero central charge $c_{2}$, two cases must be distinguished:

1. If $p$ belongs to the orbit of a constant $p(\phi)=k \in \mathbb{R}$, two qualitatively different situations may occur:

- for $k=-n^{2} c_{2} / 48 \pi$ with $n \in \mathbb{N}^{*}$, the little algebra is three-dimensional and is generated by the vector fields $\left\{\frac{\partial}{\partial \phi}, \sin n \phi \frac{\partial}{\partial \phi}, \cos n \phi \frac{\partial}{\partial \phi}\right\}$, so that $\mathfrak{g}_{k, c_{2}} \cong$ $\mathfrak{s l}^{(n)}(2, \mathbb{R})$;

- for $k \neq-n^{2} c_{2} / 48 \pi$ with $n \in \mathbb{N}^{*}$, the little algebra is one-dimensional, $\mathfrak{g}_{k, c_{2}} \cong$ $\mathfrak{u}(1)$, and is generated by $\frac{\partial}{\partial \phi}$. 
2. If $p$ does not belong to the orbit of a constant, there are again two families of solutions:

- either $\mathfrak{g}_{p, c_{2}}$ is a one-dimensional subalgebra of $\operatorname{Vect}\left(S^{1}\right)$ with solutions $X$ having finitely many simple zeros;

- or $\mathfrak{g}_{p, c_{2}}$ is a one-dimensional subalgebra of $\operatorname{Vect}\left(S^{1}\right)$ with solutions $X$ having finitely many double zeros.

\subsection{Orbits and little groups}

The little group $G_{p}$ of $p$ (at fixed central charge $c_{2}$ ) consists of all diffeomorphisms $f$ that leave $p$ fixed:

$$
p(f(\phi)) f^{\prime}(\phi)^{2}-\frac{c_{2}}{24 \pi} S[f](\phi)=p(\phi),
$$

in accordance with (4.16). The corresponding orbit $\mathcal{O}_{p}$ is diffeomorphic to the following coset spaces: ${ }^{3}$

$$
\mathcal{O}_{p} \cong \widetilde{\operatorname{Diff}^{+}}\left(S^{1}\right) / \widetilde{G}_{p} \cong \operatorname{Diff}^{+}\left(S^{1}\right) / G_{p}
$$

with $\widetilde{G}_{p}:=\chi^{-1}\left(G_{p}\right)$, where $\chi$ is the projection defined in (4.8).

For vanishing central charge $c_{2}$, the little groups are determined by the set $Z$ of zeros of $p$ :

1. If $Z=\varnothing, p$ belongs to the orbit of the constant $k$ given by $2 \pi \sqrt{|k|}=\int_{0}^{2 \pi} d \phi|p(\phi)|^{\frac{1}{2}} \neq$ 0 , whose sign is the same as that of $p$. The corresponding little group is the group $\mathrm{U}(1)$ of rigid rotations.

2. If $Z \neq \varnothing$ and $\operatorname{int} Z=\varnothing$, the little group is a finite cyclic subgroup of $\operatorname{Diff}^{+}\left(S^{1}\right)$.

3. If $Z \neq \varnothing$ and int $Z \neq \varnothing$, the little group is an infinite-dimensional subgroup of $\operatorname{Diff}^{+}\left(S^{1}\right)$.

For non-zero central charge $c_{2}$, the classification is more involved. It can be carried out through the analysis of the monodromy matrix $M_{\psi}$ of Hill's equation for the function $\psi$, with a potential proportional to $p(\phi)$. Here we will mostly state the results without proof, except for a few explicit computations in the simplest cases. A complete discussion, including proofs, can be found for instance in $[69,72]$. We assume $c_{2}>0$; the case $c_{2}<0$ follows by changing the sign of $p$.

Orbits with constant representatives. There are four families of orbits that admit a constant representative $p=k \in \mathbb{R}$ :

1. For $k=-n^{2} c_{2} / 48 \pi$ with $n \in \mathbb{N}^{*}$, the little group is $G_{n}:=\operatorname{PSL}^{(n)}(2, \mathbb{R})$, the $n$ fold cover of $\operatorname{PSL}(2, \mathbb{R})$. Indeed, in that case, the stationarity condition (6.2) for $f$ reduces to

$$
S[f]=\frac{n^{2}}{2}\left(1-\left(f^{\prime}\right)^{2}\right) .
$$

\footnotetext{
${ }^{3}$ The notation without tilde's refers to the definition of $\widehat{\mathrm{BMS}}_{3}$ in terms of the diffeomorphism group instead of its universal cover.
} 
This can be written as $\bar{S}_{n}[f]=0$ and is thus equivalent to $S\left[t_{n} \circ f \circ t_{n}^{-1}\right](x)=0$, implying that $t_{n} \circ f \circ t_{n}^{-1}(x)=(a x+b) /(c x+d)$ with $a d-b c=1$; the function $t_{n}$ is the one introduced above (4.14). This yields

$$
e^{i n f(\phi)}=\frac{\alpha e^{i n \phi}-\beta}{-\bar{\beta} e^{i n \phi}+\bar{\alpha}}, \quad\left(\begin{array}{cc}
\alpha & -\beta \\
-\bar{\beta} & \bar{\alpha}
\end{array}\right) \in \mathrm{SU}(1,1),
$$

where $\alpha, \beta$ are given by (5.9) in terms of $a, b, c, d$. (The comparison with (5.10) amounts to the transformation mapping $M \in \mathrm{SL}(2, \mathbb{R})$ on $\left(M^{-1}\right)^{T}$.) Furthermore, the associated function $\theta$ defined in (4.9) is given by

$$
\theta(\phi)=\sqrt{1+4|\alpha \beta|^{2}}-2|\alpha \beta| \cos \left(n \phi-\phi_{0}\right),
$$

with $\phi_{0}:=\arg \alpha+\arg \beta+\pi$. In this case, the monodromy matrix $M_{\psi}$ turns out to be of the form $(-)^{n} \mathbb{I}$, where $\mathbb{I}$ denotes the $2 \times 2$ identity matrix.

2. For $k<0$ but $k \neq-n^{2} c_{2} / 48 \pi$ with $n \in \mathbb{N}^{*}$, the stationarity condition still gives rise to equations (6.3), (6.4) and (6.5), now with $n$ replaced by $\sqrt{-48 \pi k / c_{2}}$. In this case, the $\theta$ function (6.5) is $2 \pi$-periodic iff $\alpha \beta=0$, implying $\beta=0$ and $f(\phi)=\phi+$ cst. The associated little group $G_{k}$ is thus isomorphic to the group $\mathrm{U}(1)$ of rigid rotations, whose universal cover is $\widetilde{G}_{k}=\mathbb{R}$. These orbits have elliptic monodromy as the associated monodromy matrix turns out to satisfy $\left|\operatorname{Tr} M_{\psi}\right|>2$.

3. For $k>0$, equations (6.3), (6.4) and (6.5) still have to hold, with $n$ replaced by $i \sqrt{48 \pi k / c_{2}}$. The same reasoning as in the previous case then implies that the corresponding little group $G_{k}$ is, once more, the group $\mathrm{U}(1)$ of rigid rotations (with $\left.\widetilde{G}_{k}=\mathbb{R}\right)$. These orbits have hyperbolic monodromy, $\left|\operatorname{Tr} M_{\psi}\right|<2$.

4. Finally, for $k=0$, the stationarity condition (6.2) reduces to $S[f]=0$, which is solved by $f=(a \phi+b) /(c \phi+d)$. However, this defines a diffeomorphism of the circle only when $c=0$, which implies that $f$ must be a rigid rotation. Hence the associated little group $G_{0}$ is again $\mathrm{U}(1)$. The corresponding monodromy matrix is of parabolic type, $\left|\operatorname{Tr} M_{\psi}\right|=2$.

Orbits without constant representatives. There are two additional families of orbits, that do not contain any constant $p(\phi)$ :

1. The orbits of the first family are labelled by the parameters $\mu>0$ and $n \in \mathbb{N}^{*}$, and have hyperbolic monodromy. An explicit representative for the $n, \mu$ orbit is given by

$$
\begin{aligned}
\frac{12 \pi p(\phi)}{c_{2}} & :=\mu^{2}+\frac{n^{2}+4 \mu^{2}}{2 F(\phi)}-\frac{3}{4} \frac{n^{2}}{F^{2}(\phi)} \\
F(\phi) & :=\cos ^{2} \frac{n \phi}{2}+\left(\sin \frac{n \phi}{2}+\frac{2 \mu}{n} \cos \frac{n \phi}{2}\right)^{2}>0 .
\end{aligned}
$$

Each such orbit can be understood as a tachyonic deformation of the orbit of the constant $-n^{2} c_{2} / 48 \pi$. The latter is indeed recovered by taking the limit $\mu \rightarrow 0$ of 
expression (6.6). The little group $G_{n, \mu}$ associated with an $n, \mu$ orbit is isomorphic to $\mathbb{R}_{+}^{*} \times \mathbb{Z}_{n}$, where $\mathbb{R}_{+}^{*}$ is the multiplicative group of positive real numbers and $\mathbb{Z}_{n}$ is the cyclic group of rigid rotations on $S^{1}$ by multiples of the angle $2 \pi / n$. Its universal cover is $\widetilde{G}_{n, \mu}=\mathbb{R} \times \mathbb{Z}$. The little algebra is generated by the vector field $X(\phi) \frac{\partial}{\partial \phi}$, with

$$
X(\phi)=\frac{1}{F(\phi)} \cos \frac{n \phi}{2}\left(\frac{2 \mu}{n} \cos \frac{n \phi}{2}+\frac{2}{n} \sin \frac{n \phi}{2}\right)
$$

which has $2 n$ simple zeros.

2. The orbits of the second family are labelled by $n \in \mathbb{N}^{*}$ and $\epsilon \in\{ \pm 1\}$ and have parabolic monodromy. An explicit representative for the $n, \epsilon$ orbit is given by

$$
\begin{aligned}
\frac{12 \pi p(\phi)}{c_{2}} & :=\frac{n^{2}}{2 H(\phi)}-\frac{3 n^{2}(1+\epsilon / 2 \pi)}{4 H^{2}(\phi)}, \\
H(\phi) & :=1+\frac{\epsilon}{2 \pi} \sin ^{2}(n \phi / 2)>0 .
\end{aligned}
$$

The corresponding little groups are, again, $G_{n, \epsilon} \cong \mathbb{R}_{+}^{*} \times \mathbb{Z}_{n}, \widetilde{G}_{n, \epsilon}=\mathbb{R} \times \mathbb{Z}$. Its Lie algebra is generated by the vector field $X(\phi) \frac{\partial}{\partial \phi}$ with

$$
X(\phi)=\frac{1}{H(\phi)} \sin ^{2}(n \phi / 2),
$$

having $n$ double zeros. Orbits of this type can be understood as massless deformations of constant orbits of the type $-n^{2} c_{2} / 48 \pi$.

\subsection{Energy bounds}

The energy of a Virasoro coadjoint vector $\left(p, i c_{2}\right)$ is defined as

$$
E_{p}:=\int_{0}^{2 \pi} d \phi p(\phi)
$$

Similarly, according to (4.16), the energy of an element $\operatorname{Ad}_{f^{-1}}^{*}\left(p, i c_{2}\right)$ belonging to the orbit $\mathcal{O}_{p}$ of $\left(p, i c_{2}\right)$ is

$$
E_{p}[f]=\int_{0}^{2 \pi} d \phi\left[\left(f^{\prime}\right)^{2} p \circ f-\frac{c}{24 \pi} S[f]\right]=\int_{0}^{2 \pi} d \phi\left[\theta p+\frac{c_{2}}{48 \pi} \frac{\left(\theta^{\prime}\right)^{2}}{\theta}\right],
$$

with $\theta$ as in (4.9). We will discuss the behaviour of energy only for $c_{2}>0$, because the discussion for $c_{2}<0$ follows from the analysis below when exchanging the words "below" and "above". (We will not consider the case $c_{2}=0$.) Since the last term of (6.8) can be made arbitrarily large by tuning $f$, it is obvious that the energy is unbounded from above on every orbit. But the real question is boundedness from below.

The inequality (4.14) readily implies that energy is bounded from below on the orbit of the constant $p(\phi)=-c_{2} / 48 \pi$ :

$$
E_{-c_{2} / 48 \pi}[f] \geqslant-c_{2} / 24
$$


with equality iff $f$ is a projective transformation of the circle as in (4.15). It also follows from the property stated after (4.14) that the global minimum of energy is reached precisely at $p=-c_{2} / 48 \pi$.

Now, for an arbitrary constant $p(\phi)=k \in \mathbb{R}$,

$$
\begin{aligned}
E_{k}[f] & =E_{-c_{2} / 48 \pi}[f]+\left(k+\frac{c_{2}}{48 \pi}\right) \int_{0}^{2 \pi} d \phi \theta \\
& =\left(E_{-c_{2} / 48 \pi}[f]+\frac{c_{2}}{24}\right)+2 \pi k+\left(k+\frac{c_{2}}{48 \pi}\right) \int_{0}^{2 \pi} d \phi\left(f^{\prime}-1\right)^{2} .
\end{aligned}
$$

Thus, provided $k>-c_{2} / 48 \pi$, the energy on the orbit $\mathcal{O}_{k}$ is bounded from below:

$$
E_{k}[f] \geqslant 2 \pi k,
$$

with equality iff $f=\phi+$ cst, the global minimum being reached at $p(\phi)=k$. To the contrary, if $k<-c_{2} / 48 \pi$, energy is unbounded from below. To see this, use a boost of rapidity $\gamma$ in the $x^{1}$ direction, realised in $\mathrm{BMS}_{3}$ through the diffeomorphism $f_{\gamma}$ given by

$$
e^{i f_{\gamma}(\phi)}=\frac{\cosh (\gamma / 2) e^{i \phi}+\sinh (\gamma / 2)}{\sinh (\gamma / 2) e^{i \phi}+\cosh (\gamma / 2)} .
$$

Such a boost preserves the energy $-c_{2} / 24$ of $-c_{2} / 48 \pi$, so it transforms the energy of $p=k$ into

$$
-\frac{c_{2}}{24}+\left(2 \pi k+\frac{c_{2}}{24}\right) \cosh \gamma
$$

When $k<-c_{2} / 48 \pi$, this can be made arbitrarily negative for sufficiently large rapidity. In conclusion, on the orbit of a constant $p(\phi)$, energy is bounded from below iff the constant representative of the orbit is situated above $-c_{2} / 48 \pi$.

As regards orbits without constant representatives, one can show that the energy is unbounded from below on all of them, except for the orbit of the massless deformation of $p=-c_{2} / 48 \pi$ with $\epsilon=-1$. In the latter case, the lower bound of energy is $-c_{2} / 24$, but it is not reached on the orbit.

\subsection{Features of $\mathrm{BMS}_{3}$ particles}

Supermomentum. The coadjoint vectors $\left(p, i c_{2}\right)$ of $\widehat{\operatorname{Vect}}\left(S^{1}\right)_{\mathrm{ab}}^{*}$ are paired with centrally extended supertranslations $(\alpha,-i b)$ according to formula (4.5) with $X$ and $a$ replaced by $\alpha$ and $b$. The tensor density $p$ should thus be interpreted as the supermomentum of a $\mathrm{BMS}_{3}$ particle since its Fourier modes are related to the supertranslation generators $P_{m}$ defined in (5.6),

$$
\left\langle\left(p, i c_{2}\right), P_{m}\right\rangle=\int_{0}^{2 \pi} d \phi p(\phi) e^{i m \phi}+\frac{c_{2}}{24} \delta_{m, 0} .
$$

In particular, the energy (6.7) of a particle with supermomentum $p$ is associated with the generator $P_{0}$ of time translations, up to a constant shift. In addition, the Fourier modes $p_{ \pm 1}$ of $p(\phi)$ encode linear momentum since they are conjugate to spatial translations.

The Virasoro coadjoint orbits listed above are thus supermomentum orbits of an appropriate $\mathrm{BMS}_{3}$ particle. From this point of view, the orbits at non-zero central charge $c_{2}$, 
on which we now focus, are similar to those of the Poincaré group: the simplest orbits with a constant non-exceptional representative are the orbits of a $\mathrm{BMS}_{3}$ particle at rest, with little group $\mathrm{U}(1)$ - exactly as for the Poincaré group. The sequence of exceptional orbits sitting at constants $-n^{2} c_{2} / 48 \pi$ looks like an infinite series of copies of the vacuum orbit in the Poincaré case, as they all have little group $\operatorname{PSL}(2, \mathbb{R})$ or a cover thereof. Finally, each of these "vacuum orbits" labelled by $n$ is accompanied by two massless orbits and a family of tachyonic orbits, which we thus take to be the orbits of massless and tachyonic $\mathrm{BMS}_{3}$ particles.

Restricting physical $\mathrm{BMS}_{3}$ particles by the criterion of boundedness of energy from below then requires these particles to have energy larger than that of the only stable vacuum orbit, the one sitting at $p=-c_{2} / 48 \pi$. The only remaining physical particles then are 1. massless particles with $\epsilon=-1$ and 2 . massive particles above $-c_{2} / 48 \pi$, the nature of which changes when the rest energy of the particle crosses $2 \pi k=0$. This qualitative change is reflected in the change of the conjugacy class of the monodromy matrix classifying these orbits.

$\mathbf{B M S}_{3}$ spin. With our definition (5.1), the $\widehat{\mathrm{BMS}}_{3}$ group is simply connected. Then the appropriate little groups are the covers $\widetilde{G}_{p}$ rather than the $G_{p}$ 's. In particular, we see from the list above that the unitary irreducible representations of each $\widetilde{G}_{p}$ involve a continuous label.

Hence, if the spin of a $\mathrm{BMS}_{3}$ particle is defined as the label of the chosen unitary irreducible representation of $\widetilde{G}_{p}$, the spin of both massive and massless particles is not quantized, just as in the three-dimensional Poincaré case. Had we defined the BMS 3 group in terms of $\operatorname{Diff}^{+}\left(S^{1}\right)$ instead of its universal cover, this would correspond to projective representations.

Further comments on induced representations of $\widehat{\mathrm{BMS}}_{3}$. In order to construct a unitary induced representation, a Virasoro-invariant measure $\mathcal{D} \mu$ on coadjoint orbits is required. The most natural way to obtain such a measure is to give a meaning to the coadjoint symplectic form $\omega$ taken to an "infinite power", yielding a volume form on the corresponding orbit. It was argued in [73] that such a construction makes sense. It is not clear to us whether this measure can indeed be used in the present context. If yes, the rest of the construction should go through: for the orbits of physical massive particles for instance, the little group is abelian and one-dimensional, and is therefore labelled by one real number $j$. In that case, the space $\mathcal{E}$ of the representation of the little group is just $\mathbb{C}$. Thus the space $\mathcal{H}$ of the induced representation of a massive particle is the space of complex-valued wavefunctionals, defined on the Virasoro coajdoint orbit of a supermomentum with rest energy $>-c_{2} / 48 \pi$, that are square-integrable with respect to the functional measure $\mathcal{D} \mu$. The representations obtained in this way are automatically unitary and irreducible, and act on one-particle states with definite supermomentum according to the formula (2.12). 


\section{Open questions}

There are a number of obvious open questions that should be addressed in the future.

1. The infinite-dimensional aspects of the problem need to be better understood, in particular questions about the existence of an invariant measure on the relevant Virasoro coadjoint orbits.

2. Provided the first issue can be solved, the next problem is to systematically study whether the inducing construction exhausts all the unitary irreducible representations of $\widehat{\mathrm{BMS}}_{3}$, along the lines of what has been achieved for the globally well-defined version of the $\mathrm{BMS}_{4}$ group.

3. A second related problem is to study the associated representations of the $\widehat{\mathfrak{b m s}}_{3}$ Lie algebra and work out those that can be related through a "flat limit" to the highestweight representations of (two copies of) the Virasoro algebra that appear in the AdS case.

4. Finally, a similar analysis should be applied to the local versions of the $\mathrm{BMS}_{4}$ group and algebra.

Note added. The Virasoro coadjoint orbits reviewed here are also a crucial ingredient in the discussion of the coadjoint representation of $\widehat{\mathrm{BMS}}_{3}$ studied in the companion paper [74]. In particular, the discussion on energy bounds can be used to derive positive energy theorems for three-dimensional gravity with non-trivial asymptotics [75], both at null infinity for the flat background and at spatial infinity for the anti-de Sitter background. While completing this and the companion paper, preprint [76] appeared, which relies on the same understanding of the role of Virasoro coadjoint orbits for the covariant phase space of asymptotically $\mathrm{AdS}_{3}$ gravity.

\section{Acknowledgments}

This work is supported in part by the Fund for Scientific Research-FNRS (Belgium), by IISN-Belgium and by "Communauté française de Belgique - Actions de Recherche Concertées". B.O. gratefully acknowledges useful discussions on related topics with Mélanie Bertelson, Cédric De Groote and Pierre-Henry Lambert.

Open Access. This article is distributed under the terms of the Creative Commons Attribution License (CC-BY 4.0), which permits any use, distribution and reproduction in any medium, provided the original author(s) and source are credited.

\section{References}

[1] G. 't Hooft, Dimensional reduction in quantum gravity, gr-qc/9310026 [INSPIRE].

[2] L. Susskind, The World as a hologram, J. Math. Phys. 36 (1995) 6377 [hep-th/9409089] [INSPIRE]. 
[3] J.M. Maldacena, The large-N limit of superconformal field theories and supergravity, Adv. Theor. Math. Phys. 2 (1998) 231 [Int. J. Theor. Phys. 38 (1999) 1113] [hep-th/9711200] [INSPIRE].

[4] A. Ashtekar and A. Magnon, Asymptotically anti-de Sitter space-times, Class. Quant. Grav. 1 (1984) L39 [INSPIRE].

[5] M. Henneaux and C. Teitelboim, Asymptotically anti-de Sitter Spaces, Commun. Math. Phys. 98 (1985) 391 [inSPIRE].

[6] M. Henneaux, Asymptotically anti-de Sitter universes in $d=3,4$ and higher dimensions, in Proceedings of the Fourth Marcel Grossmann Meeting on General Relativity, Rome Italy (1985), R. Ruffini eds., Elsevier Science Publishers B.V., Amsterdam Netherlands (1986), pg. 959.

[7] A. Ashtekar and S. Das, Asymptotically Anti-de Sitter space-times: Conserved quantities, Class. Quant. Grav. 17 (2000) L17 [hep-th/9911230] [INSPIRE].

[8] J.D. Brown and M. Henneaux, Central charges in the canonical realization of asymptotic symmetries: an example from three-dimensional gravity, Commun. Math. Phys. 104 (1986) 207 [InSPIRE].

[9] A.A. Belavin, A.M. Polyakov and A.B. Zamolodchikov, Infinite conformal symmetry in two-dimensional quantum field theory, Nucl. Phys. B 241 (1984) 333 [InSPIRE].

[10] H. Bondi, M.G.J. van der Burg and A.W.K. Metzner, Gravitational waves in general relativity. 7. Waves from axisymmetric isolated systems, Proc. Roy. Soc. Lond. A 269 (1962) 21 [inSPIRE].

[11] R.K. Sachs, Gravitational waves in general relativity. 8. Waves in asymptotically flat space-times, Proc. Roy. Soc. Lond. A 270 (1962) 103 [InSPIRE].

[12] R. Sachs, Asymptotic symmetries in gravitational theory, Phys. Rev. 128 (1962) 2851 [INSPIRE].

[13] A. Strominger, On BMS Invariance of Gravitational Scattering, arXiv:1312.2229 [INSPIRE].

[14] A. Strominger, Asymptotic Symmetries of Yang-Mills Theory, arXiv:1308.0589 [INSPIRE].

[15] T. He, V. Lysov, P. Mitra and A. Strominger, BMS supertranslations and Weinberg's soft graviton theorem, arXiv:1401.7026 [INSPIRE].

[16] T. Banks, The Super BMS Algebra, Scattering and Holography, arXiv:1403.3420 [INSPIRE].

[17] C. Duval, G.W. Gibbons and P.A. Horvathy, Conformal Carroll groups and BMS symmetry, Class. Quant. Grav. 31 (2014) 092001 [arXiv: 1402.5894] [INSPIRE].

[18] E.T. Newman, A possible connexion between the gravitational field and elementary particle physics, Nature 206 (1965) 811.

[19] A. Komar, Quantized gravitational theory and internal symmetries, Phys. Rev. Lett. 15 (1965) 76 [InSPIRE].

[20] P.J.M. Mccarthy, Asymptotically flat space-times and elementary particles, Phys. Rev. Lett. 29 (1972) 817 [INSPIRE].

[21] G. Barnich and C. Troessaert, Symmetries of asymptotically flat 4 dimensional spacetimes at null infinity revisited, Phys. Rev. Lett. 105 (2010) 111103 [arXiv:0909.2617] [InSPIRE]. 
[22] G. Barnich and C. Troessaert, Aspects of the BMS/CFT correspondence, JHEP 05 (2010) 062 [arXiv: 1001.1541] [INSPIRE].

[23] G. Barnich and C. Troessaert, Comments on holographic current algebras and asymptotically flat four dimensional spacetimes at null infinity, JHEP 11 (2013) 003 [arXiv:1309.0794] [INSPIRE].

[24] A. Ashtekar, J. Bicak and B.G. Schmidt, Asymptotic structure of symmetry reduced general relativity, Phys. Rev. D 55 (1997) 669 [gr-qc/9608042] [INSPIRE].

[25] G. Barnich and G. Compere, Classical central extension for asymptotic symmetries at null infinity in three spacetime dimensions, Class. Quant. Grav. 24 (2007) F15 [gr-qc/0610130] [INSPIRE].

[26] O. Coussaert, M. Henneaux and P. van Driel, The asymptotic dynamics of three-dimensional Einstein gravity with a negative cosmological constant, Class. Quant. Grav. 12 (1995) 2961 [gr-qc/9506019] [INSPIRE].

[27] A. Strominger, Black hole entropy from near horizon microstates, JHEP 02 (1998) 009 [hep-th/9712251] [INSPIRE].

[28] M. Bañados, Three-dimensional quantum geometry and black holes, hep-th/9901148 [INSPIRE].

[29] K. Skenderis and S.N. Solodukhin, Quantum effective action from the AdS /CFT correspondence, Phys. Lett. B 472 (2000) 316 [hep-th/9910023] [INSPIRE].

[30] G. Barnich and C. Troessaert, Supertranslations call for superrotations, PoS (CNCFG2010) 010 [arXiv: 1102.4632] [INSPIRE].

[31] G. Barnich, A. Gomberoff and H.A. González, Flat limit of three dimensional asymptotically anti-de Sitter spacetimes, Phys. Rev. D 86 (2012) 024020 [arXiv: 1204.3288] [INSPIRE].

[32] G. Barnich, Entropy of three-dimensional asymptotically flat cosmological solutions, JHEP 10 (2012) 095 [arXiv: 1208.4371] [INSPIRE].

[33] A. Bagchi, S. Detournay, R. Fareghbal and J. Simon, Holography of 3d Flat Cosmological Horizons, Phys. Rev. Lett. 110 (2013) 141302 [arXiv: 1208.4372] [INSPIRE].

[34] G. Barnich, A. Gomberoff and H.A. González, Three-dimensional Bondi-Metzner-Sachs invariant two-dimensional field theories as the flat limit of Liouville theory, Phys. Rev. D 87 (2013) 124032 [arXiv:1210.0731] [INSPIRE].

[35] G. Barnich and H.A. González, Dual dynamics of three dimensional asymptotically flat Einstein gravity at null infinity, JHEP 05 (2013) 016 [arXiv:1303.1075] [INSPIRE].

[36] G.W. Mackey, Imprimitivity for Representations of Locally Compact Groups I, Proc. Nat. Acad. Sci. U.S.A. 35 (1949) 537.

[37] G.W. Mackey, Induced representations of locally compact groups. I, Ann. Math. 55 (1952) 101.

[38] G.W. Mackey, Induced representations of locally compact groups. II. The Frobenius reciprocity theorem, Ann. Math. 58 (1953) 193.

[39] G. Mackey, Induced representations of groups and quantum mechanics, Publicazioni della Classe di Scienze della Scuola Normale Superiore di Pisa, W.A. Benjamin, New York U.S.A. (1968). 
[40] A.O. Barut and R. Raczka, Theory of Group Representations and Applications, Polish Scientific Publishers, Warszawa Poland (1980).

[41] J.F. Cornwell, Group theory in physics. vol. 1, Techniques Of Physics 7, Academic Press, London U.K. (1984), pg. 399.

[42] J.F. Cornwell, Group theory in physics. vol. 2, Techniques Of Physics 7, Academic Press, London U.K. (1984), pg. 399.

[43] E.P. Wigner, On Unitary Representations of the Inhomogeneous Lorentz Group, Annals Math. 40 (1939) 149 [INSPIRE].

[44] R.P. Geroch and E.T. Newman, Application of the semidirect product of groups, J. Math. Phys. 12 (1971) 314 [INSPIRE].

[45] P.J. McCarthy, Structure of the Bondi-Metzner-Sachs Group, J. Math. Phys. 13 (1972) 1837.

[46] P.J. McCarthy, Representations of the Bondi-Metzner-Sachs Group. I. Determination of the Representations, Proc. Roy. Soc. Lond. A 330 (1972) 517.

[47] P.J. McCarthy, Representations of the Bondi-Metzner-Sachs Group. II. Properties and Classification of the Representations, Proc. Roy. Soc. Lond. A 333 (1973) 317.

[48] P.J. McCarthy and M. Crampin, Representations of the Bondi-Metzner-Sachs Group. III. Poincare Spin Multiplicities and Irreducibility, Proc. Roy. Soc. Lond. A 335 (1973) 301.

[49] P.J. McCarthy, The Bondi-Metzner-Sachs Group in the Nuclear Topology, Proc. Roy. Soc. Lond. A 343 (1975) 489.

[50] P.J. McCarthy, Hyperfunctions and Asymptotic Symmetries, Proc. Roy. Soc. Lond. A $\mathbf{3 5 8}$ (1978) 495.

[51] P. McCarthy, Lifting of projective representations of the Bondi-Metzner-Sachs group, Proc. Roy. Soc. Lond. A 358 (1978) 141.

[52] A. Piard, Unitary Representations of Semidirect Product Groups with Infinite Dimensional Abelian Normal Subgroup, Rept. Math. Phys. 11 (1977) 259 [INSPIRE].

[53] A. Piard, Representations of the Bondi-Metzner-Sachs Group with the Hilbert Topology, Rept. Math. Phys. 11 (1977) 279 [InSPIRE].

[54] A.A. Kirillov, Lectures on the orbit method, American Mathematical Society, Providence U.S.A. (2004).

[55] V. Lazutkin and T. Pankratova, Normal forms and versal deformations for Hill's equation, Funkts. Anal. Prilozh. 9 (1975) 41.

[56] G. Segal, Unitarity Representations of Some Infinite Dimensional Groups, Commun. Math. Phys. 80 (1981) 301 [inSPIRE].

[57] E. Witten, Coadjoint Orbits of the Virasoro Group, Commun. Math. Phys. 114 (1988) 1 [INSPIRE].

[58] I. Bakas, Conformal Invariance, the KdV Equation and Coadjoint Orbits of the Virasoro Algebra, Nucl. Phys. B 302 (1988) 189 [INSPIRE].

[59] W. Taylor, Virasoro representations on diff S1/S1 coadjoint orbits, hep-th/9204091 [INSPIRE].

[60] M. Henkel, R. Schott, S. Stoimenov and J. Unterberger, On the dynamical symmetric algebra of ageing: Lie structure, representations and Appell systems, math/0510096 [INSPIRE]. 
[61] A. Bagchi and R. Gopakumar, Galilean Conformal Algebras and AdS/CFT, JHEP 07 (2009) 037 [arXiv: 0902.1385] [INSPIRE].

[62] A. Hosseiny and S. Rouhani, Affine Extension of Galilean Conformal Algebra in 2+1 Dimensions, J. Math. Phys. 51 (2010) 052307 [arXiv: 0909.1203] [INSPIRE].

[63] A. Bagchi, R. Gopakumar, I. Mandal and A. Miwa, GCA in 2d, JHEP 08 (2010) 004 [arXiv:0912.1090] [INSPIRE].

[64] V. Bargmann, On Unitary ray representations of continuous groups, Annals Math. 59 (1954) 1 [INSPIRE].

[65] D. Simms, Lecture Notes in Mathematics. Vol. 52: Lie Groups and Quantum Mechanics, Springer, Berlin Germany (1968).

[66] S. Weinberg, The Quantum Theory of Fields. Vol. 1: Foundations, Cambridge University Press, Cambidge U.K. (1995).

[67] B. Binegar, Relativistic Field Theories in Three-dimensions, J. Math. Phys. 23 (1982) 1511 [INSPIRE].

[68] V. Ovsienko and S. Tabachnikov, Projective Differential Geometry Old and New. From the Schwarzian Derivative to Cohomology of Diffeomorphism Groups, Cambridge University Press, Cambridge U.K. (2005).

[69] L. Guieu and C. Roger, L'Algèbre et le Groupe de Virasoro, Les Publications CRM, Montréal Canada (2007).

[70] B. Khesin and R. Wendt, The Geometry of Infinite-Dimensional Groups, Springer-Verlag, Heidelberg Germany (2009).

[71] J. Unterberger and C. Roger, The Schrödinger-Virasoro Algebra: Mathematical structure and dynamical Schrödinger symmetries, Springer, Heidelberg Germany (2012).

[72] J. Balog, L. Feher and L. Palla, Coadjoint orbits of the Virasoro algebra and the global Liouville equation, Int. J. Mod. Phys. A 13 (1998) 315 [hep-th/9703045] [INSPIRE].

[73] J. Dai and D. Pickrell, The orbit method and the Virasoro extension of Diff+(S1): I. Orbital integrals, J. Geom. Phys. 44 (2003) 623.

[74] G. Barnich and B. Oblak, Notes on the BMS group in three dimensions: II. Coadjoint representation, to appear (2014).

[75] G. Barnich and B. Oblak, Holographic positive energy theorems in three-dimensional gravity, arXiv: 1403.3835 [INSPIRE].

[76] A. Garbarz and M. Leston, Classification of Boundary Gravitons in $A d S_{3}$ Gravity, arXiv: 1403.3367 [INSPIRE]. 